\title{
Risk factors for preeclampsia and eclampsia at a main referral maternity hospital in Freetown, Sierra Leone: a case-control study
}

N. Stitterich ${ }^{1 *}$ (D, J. Shepherd ${ }^{2}$, M. M. Koroma ${ }^{3}$ and S. Theuring ${ }^{1}$

\begin{abstract}
Background: In the African region, 5.6\% of pregnancies are estimated to be complicated by preeclampsia and $2.9 \%$ by eclampsia, with almost one in ten maternal deaths being associated with hypertensive disorders. In Sierra Leone, representing one of the countries with the highest maternal mortality rates in the world, $16 \%$ of maternal deaths were caused by pregnancy-induced hypertension in 2016. In the light of the high burden of preeclampsia and eclampsia (PrE/E) in Sierra Leone, we aimed at assessing population-based risk factors for PrE/E to offer improved management for women at risk.
\end{abstract}

Methods: A facility-based, unmatched observational case-control study was conducted in Princess Christian Maternity Hospital (PCMH). PCMH is situated in Freetown and is the only health care facility providing 'Comprehensive Emergency Obstetric and Neonatal Care services' throughout the entire country. Cases were defined as pregnant or postpartum women diagnosed with PrE/E, and controls as normotensive postpartum women. Data collection was performed with a questionnaire assessing a wide spectrum of factors influencing pregnant women's health. Statistical analysis was performed by estimating a binary logistic regression model.

Results: We analyzed data of 672 women, 214 cases and 458 controls. The analysis yielded several independent predictors for PrE/E, including family predisposition for PrE/E (AOR $=2.72,95 \% \mathrm{Cl}: 1.46-5.07)$, preexisting hypertension ( $A O R=3.64,95 \% \mathrm{Cl}: 1.32-10.06)$, a high mid-upper arm circumflex ( $\mathrm{AOR}=3.09,95 \% \mathrm{Cl}: 1.83-5.22$ ), presence of urinary tract infection during pregnancy (AOR $=2.02,95 \% \mathrm{Cl}: 1.28-3.19)$, presence of prolonged diarrhoea during pregnancy ( $A O R=2.81,95 \% \mathrm{Cl}: 1.63-4.86)$, low maternal assets ( $\mathrm{AOR}=2.56,95 \% \mathrm{Cl}: 1.63-4.02)$, inadequate fruit intake $(\mathrm{AOR}=2.58,95 \% \mathrm{Cl}: 1.64-4.06)$, well or borehole water as the main source of drinking water $(A O R=2.05,95 \% \mathrm{Cl}: 1.31-3.23)$ and living close to a waste deposit ( $\mathrm{AOR}=1.94,95 \% \mathrm{Cl}: 1.15-3.25)$.

\footnotetext{
* Correspondence: nadine.stitt@gmail.com

${ }^{1}$ Institute of Tropical Medicine and International Health,

Charité-Universitätsmedizin Berlin, corporate member of Freie Universität

Berlin, Humboldt-Universität zu Berlin, and Berlin Institute of Health,

Augustenburger Platz 1, 13353 Berlin, Germany

Full list of author information is available at the end of the article
}

(c) The Author(s). 2021 Open Access This article is licensed under a Creative Commons Attribution 4.0 International License, which permits use, sharing, adaptation, distribution and reproduction in any medium or format, as long as you give appropriate credit to the original author(s) and the source, provide a link to the Creative Commons licence, and indicate if changes were made. The images or other third party material in this article are included in the article's Creative Commons licence, unless indicated otherwise in a credit line to the material. If material is not included in the article's Creative Commons licence and your intended use is not permitted by statutory regulation or exceeds the permitted use, you will need to obtain permission directly from the copyright holder. To view a copy of this licence, visit http://creativecommons.org/licenses/by/4.0/ The Creative Commons Public Domain Dedication waiver (http://creativecommons.org/publicdomain/zero/1.0/) applies to the data made available in this article, unless otherwise stated in a credit line to the data. 
Conclusion: Our findings suggest that systematic assessment of identified PrE/E risk factors, including a family predisposition for PrE/E, preexisting hypertension, or obesity, should be performed early on in ANC, followed by continued close monitoring of first signs and symptoms of PrE/E. Additionally, counseling on nutrition, exercise, and water safety is needed throughout pregnancy as well as education on improved hygiene behavior. Further research on sources of environmental pollution in Freetown is urgently required.

Keywords: Preeclampsia, Eclampsia, Risk factors, Freetown, Sierra Leone

\section{Background}

Preeclampsia is defined as pregnancy-induced hypertension, occurring after 20 weeks of gestation, accompanied by new-onset proteinuria, maternal organ- or uteroplacental dysfunction [1]. Maternal organ dysfunction can manifest in symptoms like epigastric pain, visual disturbance, or severe headache. Uteroplacental dysfunction can lead to fetal growth restriction with low birth weight infants [1]. The complication of a generalized seizure in a preeclamptic woman is referred to as eclampsia and can lead to maternal death [2]. In the African region, $5.6 \%$ of pregnancies are estimated to be complicated by preeclampsia and $2.9 \%$ by eclampsia and hypertensive disorders in pregnant women are responsible for almost one in ten maternal deaths [3, 4]. While the etiology of preeclampsia and eclampsia $(\mathrm{PrE} / \mathrm{E})$ is still not fully understood, knowledge of specific risk factors for PrE/E in a selected healthcare setting is essential to identify women at risk. Established risk factors for PrE/E include nulliparity, multifetal gestation, previous abort/stillbirth, a history of $\operatorname{PrE} / \mathrm{E}$ as well as a family predisposition of $\operatorname{PrE} / \mathrm{E}$ and co-morbidities like chronic hypertension, pregestational diabetes, obesity, and urinary tract infections (UTIs) [5-10]. Considering large differences in living conditions, risk factors for PrE/E referred to by low- and middle-income countries as from the sub-Saharan African region can partly diverge from those from high-income countries. In sub-Saharan African countries, low educational levels were described as risk factors for PrE/E, and in a secondary analysis of a multi-country survey conducted by the World Health Organization (WHO), adolescent pregnancies significantly raised the risk of $\mathrm{PrE} / \mathrm{E}$ [11-13]. In the Democratic Republic of Congo (DRC), Nigeria and Ghana, environmental pollution with heavy metals as well as psychosocial factors like emotional stress, poor sleeping quality and the intake of traditional treatment during pregnancy increased the risk of PrE/E [13-18]. In malariaendemic countries like Central Sudan and Senegal, Plasmodium falciparum-infected placenta was described to influence the risk of PrE/E [19-21]. In Zimbabwe and Ethiopia, obesity measured with a high mid-upper arm circumflex (MUAC) heightened the risk for preeclampsia, while a regular intake of fruits and vegetables reduced the risk [22-26]. Sierra Leone is a resource-restricted West-African country with an estimated population of seven million, with Freetown as its capital [27]. In 2017, Sierra Leone reported one of the highest maternal mortality ratios in the world with 1120 maternal deaths in 100,000 live births [28]. In 2016, pregnancy-induced hypertension caused $16 \%$ of maternal deaths in Sierra Leone [29]. A recent multicenter study reporting from 10 low and middle-income countries described the incidence of eclampsia in Freetown as the highest of all study sites with 142 cases per 10,000 births and a case fatality rate of 15.5 per 10,000 births [30]. Although the burden of PrE/E in Sierra Leone is high, annual nationwide documentation and population-based risk factors for $\operatorname{PrE} / \mathrm{E}$ are not available yet. Lacking risk factor assessment makes it impossible to target specific at-risk groups and improve their health outcomes by early monitoring and case management. Therefore, our study aimed to explore the potential risk factors for PrE/E within a cohort from a tertiary care facility located in Freetown, Sierra Leone.

\section{Methods}

\section{Design and setting}

We conducted a facility-based, unmatched observational case-control study among pregnant and postpartum women attending inpatient care or skilled delivery at Princess Christian Maternity Hospital (PCMH). PCMH is the main referral maternity hospital for the entire country as well as a teaching hospital for the University of Sierra Leone, situated in Freetown. PCMH is the only health care facility providing 'Comprehensive Emergency Obstetric and Neonatal Care services' throughout the Western Area representing 21\% of the entire country population [31]. In 2018, 18.8\% of major direct obstetric emergencies in $\mathrm{PCMH}$ and $14.7 \%$ of all maternal deaths were related to PrE/E [31]. Outcome indicators of our study included primarily population-based risk factors for PrE/E and secondarily clinical presentation and birth outcomes associated with $\mathrm{PrE} / \mathrm{E}$.

\section{Recruitment and study procedures}

Between November 2018 and February 2019, pregnant and postpartum clients of PCMH were recruited according to the following eligibility criteria: A 'case' was defined as a pregnant or postpartum woman diagnosed with preeclampsia or eclampsia following National Protocols and Guidelines for Emergency Obstetric and 
Newborn Care published by the Ministry of Health of the Government of Sierra Leone in 2018 [32]. Those specify preeclampsia as a new onset of blood pressure $\geq$ $140 / 90 \mathrm{mmHg}$ during at least two readings measured four hours apart at a time $>20$ weeks of gestation with adding any of the following signs or symptoms: proteinuria of at least 2+ in urinalysis, headache, visual disturbance or generalized edema on hands or face. Eclampsia is defined as a complication of preeclampsia marked by generalized seizures and can be diagnosed without the elevated blood pressure and proteinuria if other conditions were ruled out in differential diagnoses, like epilepsy, cerebral malaria, meningitis, or hypoglycemia [32]. A 'control' was defined as a normotensive postpartum woman, who was not diagnosed with PrE/E at any time in this pregnancy. All study participants gave written consent and received detailed information about PrE/E as well as financial compensation. In agreement with the hospital administration of PCMH and the Ethical Committee of the Ministry of Health and Sanitation, Sierra Leone and Charité- Universitätsmedizin Berlin, Germany we included minors from 15 years of age onwards if they gave written consent. Regarding minors below 15 years of age we additionally obtained the consent of their present legal guardian. Cases were consecutively enrolled until the required sample size was obtained. For each case, two controls were enrolled on the same day.

\section{Data collection and statistical analysis}

Data collection was performed in face-to-face one-hour interviews based on a 66-item questionnaire conducted by four trained local midwives. The questionnaire had been developed exclusively for our study purpose and setting and was adjusted after a test period of one week. Information collected through the questionnaire covered sociodemographic, obstetric, medical, nutritional, environmental, and behavioral characteristics as well as diagnostic information and birth outcomes (see Additional Files 1 and 2). Sociodemographic characteristics included age, religion, ethnicity, relationship status, education, occupation, maternal assets, place of residence, travel time and costs to hospital, number of children, and economic status. The economic status was assessed with a nine-point score. Obstetric characteristics asked for were gravidity, previous spontaneous abortions, and stillbirths as well as induced abortions, interpregnancy interval, multifetal gestation, first pregnancy with current partner, an obstetric history of PrE/ $\mathrm{E}$, family predisposition for $\operatorname{PrE} / \mathrm{E}$, and the number of antenatal care (ANC) visits. Medical characteristics assessed were information on prolonged diarrhoea defined as a duration of $\geq 2$ weeks, UTI, anemia, gestational diabetes, placenta previa, and Malaria during pregnancy. Furthermore, we acquired information about preexisting hypertension, family predisposition of hypertension, pregestational and gestational diabetes, and family predisposition of diabetes as well as constitutional variables like body weight, and visual evidence of overweight. We limited family predisposition on first-degree relatives meaning mother, father, or full siblings. Preexisting hypertension was defined as high blood pressure diagnosed before the 20th gestational week. The MUAC was measured by study nurses on the woman's mid-upper left arm (the right arm if she was left-handed). We defined an obese pre-pregnancy body size with a MUAC $>32 \mathrm{~cm}$ relating to Okereke et al., who defined a MUAC of $33 \mathrm{~cm}$ as a reliable cut-off point for obesity in Nigeria [33]. The visual evidence of overweight described a subjective appraisal of the attending study nurse regarding the excess bodyweight of the participant. Nutritional characteristics assessed were calcium sources in the daily diet as well as the frequency of intake of fruits, vegetables, meat, and animal products, which we categorized as an adequate intake defined as weekly or daily or an inadequate intake defined as every 2 weeks, monthly or never. Environmental characteristics included sources of pollution like living close to a main road, waste deposit, oil refinery, or cooking with open fire in a closed room, working with chemicals or other sources. We also assessed the source of which women mainly drew their drinking water regarding the poorly managed water supply system in Sierra Leone [34]. Behavioral characteristics consisted of consumption of alcohol, smoking behavior, heavy physical work during pregnancy, sleep duration and quality, emotional stress, as well as visits to a traditional healer and receiving traditional treatment. There was no follow-up of study participants, except for retrieving data of subsequent birth outcomes among the pregnant cases from available patient charts. Birth outcomes included low birth weight defined as below $2500 \mathrm{~g}$, and preterm birth was defined as a fetus born prior to the 37th postmenstrual week. Questionnaires were stored in a safe in PCMH which was only accessible for one member of the study team. All data were entered into an Excel Database in anonymized form and continuously crosschecked. IBM SPSS statistical software package version 25.0 (IBM, Armonk, NY, USA) was used to perform the statistical analysis. Categorical variables were expressed as frequencies with percentages. Metric variables were expressed as mean with standard deviation in case of a normal distribution or median and interquartile ranges in case of skewed distribution. For univariate analysis with categorical variables, we used Pearson's Chi-square Test. Metric variables were compared using Independent $\mathrm{T}$-Test and Mann-Whitney U-Test. Binary logistic regression analysis was performed to identify independent risk factors. Criteria for inclusion were a $p$-value $\leq 0.001$ and odds ratio $>1.5$ in univariate analysis. Variables that were additionally considered with a $p$-value $<0.05$ were relationship status, first pregnancy with current partner, animal product intake, snoring, and Malaria. We additionally included Malaria as a 
risk factor because of the previously described strong influence of Plasmodium falciparum-infected placenta on PrE/E [19-21]. We did not consider relationship status, animal product intake, and snoring as that variables did not prove to have a significant influence in previous literature. Regarding the variable first pregnancy with current partner, we did not take it into account because of the potential risk of being confounded by for example the age of the woman [35]. Before analysis, questions with a very low response rate of $\geq 50 \%$ of missing values like current weight, smoking behavior, alcohol consumption, calcium sources in the daily diet as well as family predisposition of diabetes and pregestational diabetes were excluded. We tried to complete variables like gestational diabetes, anemia during pregnancy, and placenta previa with patient charts which turned out to be not reliable due to lacking data entries. Further variables with missing data were handled by employing multiple imputation to include all 672 study participants into a binary logistic regression model. After analyzing the missing data patterns of 47 eligible variables, we identified 23 variables that had $\geq 1 \%$ missing data. We excluded two variables, interpregnancy interval' and 'obstetric history of $\mathrm{PrE} / \mathrm{E}$ ' because missing data exceeded $30 \%$ due to a high rate of nulliparous women in our sample. As there were no patterns in the missing data of the remaining 21 variables, we chose them for imputation. Variable chosen for imputation in descending order of quantity of missing values were family predisposition for $\mathrm{PrE} / \mathrm{E}$ of all female family members, family predisposition for $\mathrm{PrE} / \mathrm{E}$ of only mother and sister, travel cost, family predisposition for chronic hypertension, living close to a main road, living close to a waste deposit, environmental pollution, maternal assets, travel time, average sleep duration, meat intake, place of residence, occupation, first pregnancy with current partner, vegetable intake, fruit intake, parity, MUAC, well or borehole water as main source of drinking water, snoring and daytime sleepiness. We added four additional variables that had $<1 \%$ of missing values but were chosen to be included in the binary logistic regression model which were Malaria, prolonged diarrhoea, UTI, and preexisting hypertension. We imputed 25 variables with 10 datasets, which raised the availability of datasets for calculation from $65.5 \%$ (440) to $100 \%$ (672). For imputation, we chose the Mersenne Twister as a random number generator program and for generator initialization, we chose the fixed value of 2,000,000. Regarding data distribution, the imputed data set showed an overall good validity. Statistical significance was set at $p$-value $<0.05$, crude and adjusted odds ratios (COR, AOR) and 95\% confidence interval (95\% CI) were calculated. The Hosmer-Lemeshow goodness of fit test for logistic regression was applied and showed overall a good fit with a $p$-value $>0.05$.

\section{Results}

A total of 697 women, 233 cases and 464 controls were recruited among pregnant and postpartum women in PCMH. Before analysis, we had to exclude 25 women who did not meet the inclusion criteria,19 cases and 6 controls. 19 cases were falsely diagnosed with $\mathrm{PrE} / \mathrm{E}$ with 17 women who did not meet national guidelines of $\mathrm{PrE} /$ $\mathrm{E}$ diagnosis because the blood pressure assessed in one reading was below $140 / 90 \mathrm{mmHg}$. One woman had complete missing diagnosis data and an eclamptic case subsequently proved to be epileptic. Out of 6 controls, 4 had episodes of gestational hypertension in pregnancy with suspected preeclampsia and 2 had an abortion before 20 weeks of pregnancy. We analyzed data from 672 women, 214 cases, and 458 controls. In the case group, $63(29.4 \%)$ women were eclamptic and two died after participation.

\section{Sociodemographic characteristics}

Women were on a mean average 25 years old, had one child, and lived by a majority of $78 \%$ in a relationship. They mainly belonged to the Muslim religion (70.8\%) and the Temne tribe (40.1\%). Almost half of the cohort were illiterate (45.1\%) and up to $20 \%$ were adolescents including 7 minors. Most women worked as selfemployed traders (46.5\%) and twice as many cases (31.6\%) were housewives compared to controls (14.1). Among postpartum recruited women, we observed an average of 5 ANC visits during their pregnancy. Women in the case group dwelled more often in a poorly equipped household $(\mathrm{COR}=1.92,95 \% \mathrm{CI}$ : 1.18-3.14) without the availability of electricity $(\mathrm{COR}=1.72,95 \%$ CI: $1.20-2.45)$ and pipe-borne water $(\mathrm{COR}=1.47,95 \%$ CI: $1.06-2.04)$. In the case group, $40.0 \%$ of women had less than 50,000 Sierra Leone Leones (SLL) of funds available per month, which equals about less than 5 USDollar $(\mathrm{COR}=2.72$, 95\% CI: 1.89-3.92). Sociodemographic differences between cases and controls are fully displayed in Table 1.

\section{Manifestations of PrE/E}

The typical symptomatic manifestation of preeclampsia in our study included headache (96.0\%), generalized edema (82.0\%), and epigastric pain (78.0\%). The majority of eclamptic cases suffered from vomiting (77.4\%) and blurred vision (67.7\%). Cases had a mean blood pressure of $161 / 109 \mathrm{mmHg}$, and $2.5+$ proteinuria in urinalysis.

\section{Univariate analysis of risk factors}

Women with $\mathrm{PrE} / \mathrm{E}$ in a previous pregnancy had an almost threefold risk for $\mathrm{PrE} / \mathrm{E}(\mathrm{COR}=2.62,95 \% \mathrm{CI}$ : 1.12-6.10), while family predisposition for PrE/E in the mother or sister even led to a fivefold risk compared to women without a family history or $\mathrm{PrE} / \mathrm{E}(\mathrm{COR}=5.33$, 
Table 1 Sociodemographic characteristics of women with and without PrE/E in PCMH

\begin{tabular}{|c|c|c|c|c|c|}
\hline Characteristics & Total n (\%) & Cases n (\%) & Controls n (\%) & COR $(95 \% \mathrm{CI})$ & $p$-value \\
\hline Total & 672 & 214 & 458 & & \\
\hline Age & $(668)$ & (213) & (455) & & \\
\hline Means & $25.30( \pm 5.19)$ & $25.01( \pm 5.95)$ & $25.43( \pm 5.91)$ & & 0.401 \\
\hline$<20$ & $128(19.2)$ & $47(22.1)$ & $81(17.8)$ & $1.33(0.88-2.02)$ & 0.171 \\
\hline $20-32$ & $442(66.2)$ & $134(62.9)$ & $308(67.7)$ & 1.0 & \\
\hline$>32$ & $98(14.7)$ & $32(15.0)$ & $66(14.5)$ & $1.11(0.70-1.78)$ & 0.650 \\
\hline Relationship ${ }^{a}$ & (668) & (211) & $(457)$ & & \\
\hline Yes & $521(78.0)$ & 175 (82.9) & $346(75.7)$ & $1.56(1.03-2.37)$ & 0.036 \\
\hline No & $147(22.0)$ & $36(17.1)$ & $111(24.3)$ & 1.0 & \\
\hline Religion & $(662)$ & (212) & $(450)$ & & \\
\hline Christian & $193(29.2)$ & $59(27.8)$ & $134(29.8)$ & 1.0 & \\
\hline Muslim & 469 (70.8) & $153(72.2)$ & $316(70.2)$ & $1.10(0.77-1.58)$ & 0.607 \\
\hline Ethnicity & (669) & (212) & $(457)$ & & \\
\hline Mende & $104(15.5)$ & $29(13.7)$ & 75 (16.4) & 1.0 & \\
\hline Temne & $268(40.1)$ & $89(42.0)$ & $179(39.2)$ & $1.29(0.78-2.12)$ & 0.322 \\
\hline Other ${ }^{\mathrm{b}}$ & $297(44.4)$ & $94(44.3)$ & $203(44.4)$ & $1.20(0.73-1.96)$ & 0.474 \\
\hline Education & (669) & (212) & $(457)$ & & \\
\hline Illiterate & $302(45.1)$ & $104(49.1)$ & $198(43.3)$ & $1.26(0.91-1.75)$ & 0.166 \\
\hline literate & 367 (54.9) & $108(50.9)$ & $259(56.7)$ & 1.0 & \\
\hline Occupation & $(662)$ & (209) & (453) & & \\
\hline Housewife & 130 (19.6) & 66 (31.6) & $64(14.1)$ & $3.88(2.43-6.22)$ & $<0.001$ \\
\hline Trader & 308 (46.5) & $96(45.9)$ & $212(46.8)$ & $1.71(1.14-2.55)$ & 0.009 \\
\hline Other $^{c}$ & 224 (33.8) & $47(22.5)$ & $177(39.1)$ & 1.00 & \\
\hline Number of Children & $(672)$ & (214) & (458) & & \\
\hline Own children & $1.01( \pm 1.20)$ & $1.08( \pm 1.24)$ & $0.98( \pm 1.17)$ & & 0.307 \\
\hline Other children & $0.33( \pm 0.71)$ & $0.27( \pm 0.68)$ & $0.36( \pm 0.72)$ & & 0.115 \\
\hline Place of residence & $(657)$ & $(207)$ & $(450)$ & & \\
\hline City & $487(74.1)$ & $151(72.9)$ & $336(74.7)$ & 1.0 & \\
\hline Suburb/Rural & $170(25.9)$ & $56(27.1)$ & $114(25.3)$ & $1.09(0.75-1.59)$ & 0.640 \\
\hline Antenatal care visits ${ }^{d}$ & (672) $5.36( \pm 2.61)$ & (214) $5.76( \pm 2.34)$ & (458) $5.18( \pm 2.70)$ & & \\
\hline Travel time ${ }^{e}$ & $(647)$ & (208) & (439) & & \\
\hline Median & $45(30-60)$ & $60(41-90)$ & $40(30-60)$ & & $<0.001$ \\
\hline Means & $52( \pm 34.91)$ & $69( \pm 38.63)$ & $45( \pm 30.02)$ & & \\
\hline Travel cost ${ }^{f}$ & $(540)$ & (194) & (346) & & \\
\hline Median & 3000 (2000-6000) & $5000(3000-8000)$ & $2750(2000-4075)$ & & $<0.001$ \\
\hline Means & $5600( \pm 8800)$ & $7600( \pm 10,400)$ & $4500( \pm 7600)$ & & \\
\hline Maternal assets ${ }^{9}$ & (647) & (205) & $(442)$ & & \\
\hline$\leq 50,000 \mathrm{SLL}$ & $169(26.1)$ & $82(40.0)$ & $87(19.7)$ & $2.72(1.89-3.92)$ & $<0.001$ \\
\hline$>50,000 \mathrm{SLL}$ & 478 (73.9) & $123(60.0)$ & $355(80.3)$ & 1.0 & \\
\hline Economic status $^{\mathrm{h}}$ & (668) & $(211)$ & $(457)$ & & \\
\hline Low & $184(27.5)$ & 75 (35.5) & 109 (23.9) & $1.92(1.18-3.14)$ & 0.008 \\
\hline Middle & $355(53.1)$ & $102(48.3)$ & $253(55.4)$ & $1.13(0.72-1.77)$ & 0.607 \\
\hline High & 129 (19.3) & $34(16.1)$ & $95(20.8)$ & 1.0 & \\
\hline
\end{tabular}


Table 1 Sociodemographic characteristics of women with and without PrE/E in PCMH (Continued)

\begin{tabular}{|c|c|c|c|c|c|}
\hline Characteristics & Total n (\%) & Cases n (\%) & Controls n (\%) & COR $(95 \% \mathrm{Cl})$ & $p$-value \\
\hline Pipe-borne water & $(667)$ & $(210)$ & $(457)$ & & \\
\hline Yes & $377(56.5)$ & $105(50.0)$ & $272(59.5)$ & 1.0 & \\
\hline No & $290(43.5)$ & $105(50.0)$ & 185 (40.5) & $1.47(1.06-2.04)$ & 0.021 \\
\hline Electricity & $(667)$ & (210) & $(457)$ & & \\
\hline Yes & $490(73.4)$ & $139(65.9)$ & $351(76.8)$ & 1.0 & \\
\hline No & 178 (26.6) & $72(34.1)$ & $106(23.2)$ & $1.72(1.20-2.45)$ & 0.003 \\
\hline \multicolumn{6}{|c|}{$\begin{array}{l}\text { Univariate analysis was conducted with Pearson's Chi-square test for categorical variables. Metric variables were compared using Independent T-Test and Mann- } \\
\text { Whitney-U-Test. Statistical significance was set at } p \text {-value }<0.05 \text {, crude and adjusted odds ratios and } 95 \% \text { confidence interval were calculated } \\
\text { a relationship status: yes = cohabit, married; no }=\text { single, divorced, widowed } \\
\text { b other ethnicities = Limba, Krio, Fullah, other } \\
\text { c other occupation = farmer, student, other } \\
\text { d excluded from univariate analysis because the variable is confounded by pregnancy status during recruitment } \\
\text { e to Princess Christian Maternity Hospital in minutes } \\
\text { f to Princess Christian Maternity Hospital in Sierra Leone Leones } \\
\text { g monthly assets; } 50,000 \text { Sierra Leone Leones equal about } 5 \text { US-Dollar } \\
\text { h economic status: electricity, radio, cattle, pipe-borne water, TV, motorbike or car, mobile phone, fridge, mosquito-net; score: low }=0-3 \text {, middle = =4-6, high }=7-9\end{array}$} \\
\hline
\end{tabular}

95\% CI: 2.66-10.69). Preexisting hypertension was significantly more often found in the case group with $7.2 \%$ of women compared to only $2.0 \%$ of women in the control group $(\mathrm{COR}=3.88$, 95\% CI: 1.67-9.01). However, family predisposition for hypertension had no significant impact on the risk of PrE/E. To have the first pregnancy with the current partner was also significantly connected to $\mathrm{PrE} / \mathrm{E}(\mathrm{COR}=1.68,95 \% \mathrm{CI}: 1.17-2.41)$. Medical conditions like UTI $(\mathrm{COR}=2.66,95 \% \mathrm{CI}: 1.82-$ $3.88)$, prolonged diarrhoea $(\mathrm{COR}=3.71$, 95\% CI: 2.49 5.53 ), and Malaria (COR $=1.60,95 \% \mathrm{CI}: 1.13-2.27)$ also significantly increased the risk for $\mathrm{PrE} / \mathrm{E}$. In the case group, more women appeared visually to be overweight (16.4\%) compared to the control group (2.0\%). In accordance with that, a high MUAC > $32 \mathrm{~cm}$ was measured in $23.9 \%$ of the case group and only $10.5 \%$ of the control group $(\mathrm{COR}=2.67,95 \% \mathrm{CI}: 1.73-4.14)$. Women in the case group proved to have a significantly lower intake of fruits $(\mathrm{COR}=3.30,95 \% \mathrm{CI}: 2.25-4.85)$ as well as animal products other than meat, while inadequate vegetable intake seemed to have a protective effect $(\mathrm{COR}=0.65,95 \%$ CI: 0.47-0.90). A short sleep duration of $<6 \mathrm{~h}$ was significantly more often observed in the case group $(\mathrm{COR}=$ 1.94, 95\% CI: $1.36-2.77)$, as well as snoring (COR $=1.82$, $95 \%$ CI: $1.18-2.79$ ). About $65 \%$ of women in our study cohort stated that they were affected by pollution in their daily living environment. In the case group, nearly twice as many women lived close to a waste deposit (29.4\%) compared to the control group (16.3\%) (COR $=2.14,95 \%$ CI: 1.44-3.18) and more cases used well or borehole water as their main source of drinking water $(\mathrm{COR}=1.90,95 \%$ CI: 1.30-2.79) (Table 2).

Multivariate analysis of independent risk factors for PrE/E Logistic regression analysis, as shown in Table 3, revealed nine independent predictors for PrE/E: a family predisposition for $\mathrm{PrE} / \mathrm{E}(\mathrm{AOR}=2.72$, 95\% CI: $1.46-$ 5.07), preexisting hypertension $(\mathrm{AOR}=3.64,95 \% \mathrm{CI}$ : 1.32-10.06), a high MUAC (AOR = 3.09, 95\% CI: $1.83-$ 5.22 ), presence of UTI during pregnancy ( $\mathrm{AOR}=2.02$, 95\% CI: 1.28-3.19), presence of prolonged diarrhoea during pregnancy $(\mathrm{AOR}=2.81,95 \% \mathrm{CI}$ : 1.63-4.86), low maternal assets $(\mathrm{AOR}=2.56,95 \% \mathrm{CI}: 1.63-4.02)$, inadequate fruit intake $(\mathrm{AOR}=2.58,95 \% \mathrm{CI}$ : 1.64-4.06), the use of well or borehole water as the main source of drinking water $(\mathrm{AOR}=2.05,95 \% \mathrm{CI}$ : $1.31-3.23)$ as well as living close to a waste deposit $(\mathrm{AOR}=1.94,95 \% \mathrm{CI}$ : 1.15-3.25). From all analyzed factors, only Malaria $(\mathrm{AOR}=1.43,95 \% \mathrm{CI}: 0.95-2.15)$ and short sleep duration $(\mathrm{AOR}=1.48,95 \% \mathrm{CI}: 0.96-2.29)$ were not independently associated with PrE/E.

\section{Birth outcomes}

Adverse birth outcomes linked to PrE/E were preterm birth $(\mathrm{COR}=4.72$, CI95\%: 3.12-7.16), low birth weight of neonates ( $\mathrm{COR}=4.67$, CI95\%: 2.81-7.76) and macerated stillbirths $(\mathrm{COR}=2.81, \mathrm{CI} 95 \%$ : 1.33-5.97). However, the overall neonatal mortality rate was not significantly higher in the case group (14.2\%) compared to the control group (9.7\%) (Table 4).

\section{Discussion}

Our study represents the first comprehensive assessment of risk factors for PrE/E in Sierra Leone. Considering the scarcity of data in our setting, we analyzed a wide spectrum of factors potentially influencing pregnant women's health. Our main findings included a preexisting hypertension, a high MUAC, presence of UTI or prolonged diarrhoea during pregnancy, family predisposition for $\mathrm{PrE} / \mathrm{E}$, inadequate fruit intake, and low maternal assets as independent risk factors for PrE/E. In addition, we explored the influence of environmental 
Table 2 Obstetrics, medical, nutritional, environmental and behavioural characteristics of women with and without PrE/E in PCMH

\begin{tabular}{|c|c|c|c|c|c|}
\hline Characteristics & Total n (\%) & Cases $\mathbf{n}(\%)$ & Controls n (\%) & COR $(95 \% \mathrm{CI})$ & $p$-value \\
\hline Gravidity & $(672)$ & $(214)$ & $(458)$ & & \\
\hline 1 & $244(36.3)$ & $84(39.3)$ & $160(34.9)$ & $1.62(1.02-2.58)$ & 0.041 \\
\hline 2 & $143(21.3)$ & $35(16.4)$ & $108(23.6)$ & 1.0 & \\
\hline$>2$ & $285(42.4)$ & $95(44.4)$ & $190(41.5)$ & $1.54(0.98-2.43)$ & 0.060 \\
\hline Parity & $(664)$ & $(212)$ & $(452)$ & & \\
\hline 0 & $258(38.9)$ & $87(41.0)$ & $171(37.8)$ & $1.14(0.82-1.60)$ & 0.429 \\
\hline$\geq 1$ & $406(61.1)$ & $125(59.0)$ & $281(62.2)$ & 1.0 & \\
\hline Previous spontaneous abort or stillbirth & $(669)$ & $(212)$ & $(457)$ & & \\
\hline Yes & $69(10.3)$ & $17(8.0)$ & $52(11.4)$ & $0.68(0.38-1.21)$ & 0.184 \\
\hline No & $600(89.7)$ & $195(92.0)$ & $405(88.6)$ & 1.0 & \\
\hline Previous induced abortion & $(671)$ & (214) & $(457)$ & & \\
\hline Yes & $46(6.9)$ & $9(4.2)$ & $37(8.1)$ & $0.50(0.24-1.05)$ & 0.063 \\
\hline No & $625(93.1)$ & $205(95.8)$ & $420(91.9)$ & 1.0 & \\
\hline Interpregnancy interval & $(411)$ & (124) & $(287)$ & & \\
\hline$\leq 18$ months & $35(8.5)$ & $13(10.5)$ & $22(7.7)$ & $1.45(0.70-2.99)$ & 0.311 \\
\hline 18-120 months & $349(84.9)$ & $101(81.5)$ & $248(86.4)$ & 1.0 & \\
\hline$>120$ months & $27(6.6)$ & $10(8.1)$ & $17(5.9)$ & $1.44(0.64-3.26)$ & 0.374 \\
\hline Multifetal gestation & $(671)$ & (213) & $(458)$ & & \\
\hline Yes & $45(6.7)$ & $18(8.5)$ & $27(5.9)$ & $1.47(0.79-2.74)$ & 0.218 \\
\hline No & $626(93.3)$ & $195(91.5)$ & $431(94.1)$ & 1.0 & \\
\hline First pregnancy with current partner & $(662)$ & $(211)$ & $(451)$ & & \\
\hline Yes & $443(66.9)$ & $157(74.4)$ & $286(63.4)$ & $1.68(1.17-2.41)$ & 0.005 \\
\hline No & $219(33.1)$ & $54(25.6)$ & $165(36.6)$ & 1.0 & \\
\hline Preeclampsia or eclampsia in obstetric history & $(407)$ & $(125)$ & $(282)$ & & \\
\hline Yes & $23(5.7)$ & $12(9.6)$ & $11(3.9)$ & $2.62(1.12-6.10)$ & 0.022 \\
\hline No & $384(94.3)$ & $113(90.4)$ & $271(96.1)$ & 1.0 & \\
\hline $\begin{array}{l}\text { Family predisposition for preeclampsia } \\
\text { and eclampsia }\end{array}$ & $(525)$ & (138) & $(387)$ & & \\
\hline Yes & $37(7.0)$ & $23(16.7)$ & $14(3.6)$ & $5.33(2.66-10.69)$ & $<0.001$ \\
\hline No & $488(93.0)$ & $115(83.3)$ & $373(96.4)$ & 1.0 & \\
\hline Preexisting hypertension & $(666)$ & (208) & $(458)$ & & \\
\hline Yes & $24(3.6)$ & $15(7.2)$ & $9(2.0)$ & $3.88(1.67-9.01)$ & 0.001 \\
\hline No & $642(96.4)$ & $193(92.8)$ & $449(98.0)$ & 1.0 & \\
\hline Family predisposition for chronic hypertension & $(612)$ & $(178)$ & $(434)$ & & \\
\hline Yes & $113(18.5)$ & $35(19.7)$ & $78(18.0)$ & $1.12(0.72-1.74)$ & 0.624 \\
\hline No & $499(81.5)$ & $143(80.3)$ & $356(82.0)$ & 1.0 & \\
\hline Prolonged diarrhoea & $(671)$ & $(214)$ & $(457)$ & & \\
\hline Yes & $127(18.9)$ & $72(33.6)$ & $55(12.0)$ & $3.71(2.49-5.53)$ & $<0.001$ \\
\hline No & $544(81.1)$ & $142(66.4)$ & $402(88.0)$ & 1.0 & \\
\hline UTI & $(670)$ & (214) & $(456)$ & & \\
\hline Yes & $145(21.6)$ & 72 (33.6) & $73(16.0)$ & $2.66(1.82-3.88)$ & $<0.001$ \\
\hline No & $525(78.4)$ & $142(66.4)$ & $383(84.0)$ & 1.0 & \\
\hline Malaria & (671) & (214) & $(457)$ & & \\
\hline Yes & $432(64.4)$ & $153(71.5)$ & $279(61.1)$ & $1.60(1.13-2.27)$ & 0.008 \\
\hline
\end{tabular}


Table 2 Obstetrics, medical, nutritional, environmental and behavioural characteristics of women with and without PrE/E in PCMH (Continued)

\begin{tabular}{|c|c|c|c|c|c|}
\hline Characteristics & Total n (\%) & Cases $\mathrm{n}(\%)$ & Controls n (\%) & COR $(95 \% \mathrm{Cl})$ & $p$-value \\
\hline No & $239(35.6)$ & $61(28.5)$ & $178(38.9)$ & 1.0 & \\
\hline Visual evidence of overweight & $(671)$ & $(213)$ & $(458)$ & & \\
\hline Yes & $44(6.6)$ & $35(16.4)$ & $9(2.0)$ & $9.95(4.67-21.19)$ & $<0.001$ \\
\hline No & $548(81.7)$ & $154(72.3)$ & $394(86.0)$ & 1.0 & \\
\hline Don't know & $79(11.8)$ & $24(11.3)$ & $55(12.0)$ & & \\
\hline MUAC & $(665)$ & (209) & $(456)$ & & \\
\hline$>32 \mathrm{~cm}$ & $98(14.7)$ & $50(23.9)$ & $48(10.5)$ & $2.67(1.73-4.14)$ & $<0.001$ \\
\hline$\leq 32 \mathrm{~cm}$ & $567(85.3)$ & $159(76.1)$ & $408(89.5)$ & 1.0 & \\
\hline Fruit intake & $(664)$ & (209) & $(455)$ & & \\
\hline Inadequate & $141(21.2)$ & $75(35.9)$ & $66(14.5)$ & $3.30(2.25-4.85)$ & $<0.001$ \\
\hline Adequate & $523(78.8)$ & $134(64.1)$ & $389(85.5)$ & 1.0 & \\
\hline Vegetable intake & $(664)$ & $(211)$ & $(453)$ & & \\
\hline Inadequate & $326(49.1)$ & $88(41.7)$ & $238(52.5)$ & $0.65(0.47-0.90)$ & 0.009 \\
\hline Adequate & $338(50.9)$ & $123(58.3)$ & $215(47.5)$ & 1.0 & \\
\hline Meat intake & $(656)$ & $(211)$ & $(445)$ & & \\
\hline Inadequate & $427(65.1)$ & $147(69.7)$ & $280(62.9)$ & $1.35(0.95-1.92)$ & 0.090 \\
\hline Adequate & $229(34.9)$ & $64(30.3)$ & $165(37.1)$ & 1.0 & \\
\hline Animal product intake & $(667)$ & $(211)$ & $(456)$ & & \\
\hline Inadequate & $253(37.9)$ & $92(43.6)$ & $161(35.3)$ & $1.42(1.02-1.98)$ & 0.040 \\
\hline Adequate & $414(62.1)$ & $119(56.4)$ & $295(64.7)$ & 1.0 & \\
\hline Environmental pollution $^{a}$ & $(631)$ & (201) & $(430)$ & & \\
\hline Yes & $408(64.7)$ & $154(76.6)$ & $254(59.1)$ & $2.27(1.55-3.32)$ & $<0.001$ \\
\hline No & $223(35.3)$ & $47(23.4)$ & $176(40.9)$ & 1.0 & \\
\hline Living close to a waste deposit & (631) & $(201)$ & $(430)$ & & \\
\hline Yes & $129(20.4)$ & $59(29.4)$ & $70(16.3)$ & $2.14(1.44-3.18)$ & $<0.001$ \\
\hline No & $502(79.6)$ & $142(70.6)$ & $360(83.7)$ & 1.0 & \\
\hline Living close to a main road & $(631)$ & (201) & $(430)$ & & \\
\hline Yes & $257(40.7)$ & $80(39.8)$ & $177(41.2)$ & $0.95(0.67-1.33)$ & 0.746 \\
\hline No & $374(59.3)$ & $121(60.2)$ & $253(58.8)$ & 1.0 & \\
\hline $\begin{array}{l}\text { Well or borehole water as main source } \\
\text { of drinking water }\end{array}$ & $(665)$ & $(211)$ & $(454)$ & & \\
\hline Yes & $141(21.2)$ & $61(28.9)$ & $80(17.6)$ & $1.90(1.30-2.79)$ & 0.001 \\
\hline No & $524(78.8)$ & $150(71.1)$ & $374(82.4)$ & 1.0 & \\
\hline Average sleep duration & $(648)$ & (198) & $(450)$ & & \\
\hline Short $<6 \mathrm{~h}$ & $386(59.6)$ & $139(70.2)$ & $247(54.9)$ & $1.94(1,36-2.77)$ & $<0.001$ \\
\hline Long $\geq 6 \mathrm{~h}$ & $262(40.4)$ & $59(29.8)$ & $203(45.1)$ & 1.0 & \\
\hline Snoring & $(665)$ & $(211)$ & $(454)$ & & \\
\hline Yes & $104(15.6)$ & $45(21.3)$ & $59(13.0)$ & $1.82(1.18-2.79)$ & 0.006 \\
\hline No & $561(84.4)$ & $166(78.7)$ & $395(87.0)$ & 1.0 & \\
\hline Heavy physical work ${ }^{b}$ & $(671)$ & $(214)$ & $(457)$ & & \\
\hline Yes & $411(61.3)$ & $113(52.8)$ & $298(65.2)$ & $0.60(0.43-0.83)$ & 0.002 \\
\hline No & $260(38.7)$ & $101(47.2)$ & 159 (34.8) & 1.0 & \\
\hline Emotional stress & $(666)$ & $(212)$ & $(454)$ & & \\
\hline Yes & $234(35.1)$ & $76(35.8)$ & $158(34.8)$ & $0.96(0.68-1.34)$ & 0.792 \\
\hline
\end{tabular}


Table 2 Obstetrics, medical, nutritional, environmental and behavioural characteristics of women with and without PrE/E in PCMH (Continued)

\begin{tabular}{|c|c|c|c|c|c|}
\hline Characteristics & Total n (\%) & Cases n (\%) & Controls n (\%) & COR $(95 \% \mathrm{Cl})$ & $p$-value \\
\hline No & $432(64.9)$ & $136(64.2)$ & $296(65.2)$ & 1.0 & \\
\hline Visit of traditional healer & $(667)$ & (214) & (453) & & \\
\hline Never & $520(78.0)$ & $167(78.0)$ & $353(77.9)$ & 1.0 & 0.705 \\
\hline Sometimes & $136(20.4)$ & $46(21.5)$ & $90(19.9)$ & $1.08(0.72-1.61)$ & 0.104 \\
\hline Often & $11(1.6)$ & $1(0.5)$ & $10(2.2)$ & $0.21(0.03-1.67)$ & \\
\hline Traditional treatment & $(667)$ & (214) & (453) & & \\
\hline Never & $462(69.3)$ & $149(69.6)$ & $313(69.1)$ & 1.0 & \\
\hline Sometimes & $174(26.1)$ & $63(29.4)$ & $111(24.5)$ & $1.19(0.83-1.72)$ & 0.345 \\
\hline Often & $31(4.6)$ & $2(0.9)$ & $29(6.4)$ & $0.15(0.03-0.62)$ & 0.003 \\
\hline
\end{tabular}

Univariate analysis was conducted with Pearson's Chi-square test for categorical variables. Metric variables were compared using Independent T-Test and MannWhitney-U-Test. Statistical significance was set at $p$-value $<0.05$, crude and adjusted odds ratios and $95 \%$ confidence interval were calculated

a environmental pollution of daily living environment: close to waste deposit, close to main road, cooking with open fire in closed room, working with chemicals e.g. pesticides, paint or car components, close to oil refinery, other sources of pollution

${ }^{b}$ heavy physical work: carry heavy weights, farm work, long distance walking ( $\geq 2 \mathrm{~h}$ per day), prolonged standing ( $\geq 6 \mathrm{~h}$ per day), manual laundry, carry heavy weights on head, other physical work

pollution on pregnant women's health and found that living close to a waste deposit as well as using well or borehole water as main source of drinking water were independently linked with $\mathrm{PrE} / \mathrm{E}$. Among those identified factors, preexisting hypertension had the strongest association with $\operatorname{PrE} / \mathrm{E}$ with an approximate fourfold increased risk. This finding corroborates a secondary analysis of a WHO Global Survey conducted in 23 low and middle-income countries, which reported that women who suffered chronic hypertension were almost 8 times more likely to become preeclamptic or eclamptic than women without the condition [9]. Chronic hypertension is linked to vascular dysfunction, which is assumed to play a key role in the pathophysiology of $\mathrm{PrE} / \mathrm{E}$ by inducing abnormal development of placental vasculature in early pregnancy, leading to disordered placental perfusion [36]. In this context, obesity is another important player, additionally fostering high blood pressure and inducing cardiovascular morbidity, which hence promotes $\mathrm{PrE} / \mathrm{E}$ [36]. Obese study participants from our cohort with a MUAC $>32 \mathrm{~cm}$ showed a threefold increased risk for PrE/E. This is supported by research from Zimbabwe, where a MUAC $\geq 28 \mathrm{~cm}$ already increased the risk for preeclampsia about 4.4 times [26], and Endeshaw et al. (2015) reported that in Ethiopia the incidence rate of preeclampsia raised by a factor of 1.35 with every $1 \mathrm{~cm}$ increase of MUAC [23]. UTIs and prolonged diarrhoea during pregnancy both doubled the risk for $\operatorname{PrE} / \mathrm{E}$ in our cohort. A systematic review investigating the association between maternal infection and preeclampsia showed that women suffering from UTIs during pregnancy were $57 \%$ more likely to develop the condition [10]. Von Dadelszen et al. (2002) suggest that inflammatory states can induce acute atherosis, causing uteroplacental perfusion insufficiency [37]. These results are consistent with findings from Bilano et al. and Kaduma et al., who reported that preeclamptic women in Tanzania were 7.7 times more likely to present with significant bacteriuria $[9,38]$. A heritable component with a family predisposition of $\mathrm{PrE} / \mathrm{E}$ in the mother or sister of a woman proved to be independently associated with a threefold higher risk for PrE/E in our analysis. In a multi-generation study accessing a birth register including 32,824 preeclamptic women, Nilsson et al. stated that a family predisposition for preeclampsia in a fullsister or mother raised a woman's risk about two to threefold, whereby an affected half-sister had no significant influence on the risk of a woman [8]. Assessing unmodifiable risk factors like the family predisposition of a pregnant woman should be a priority in the first ANC visit and enable women at risk to get access to closer monitoring and education on early signs and symptoms of PrE/E. With this study being situated in the urban setting of Freetown, $65 \%$ of women in our cohort stated to be affected by environmental pollution; living close to a waste deposit doubled the risk for PrE/E in our setting. Two recent studies from the DRC and a study from Nigeria emphasize that heavy metal pollution could raise the risk for preeclampsia; in those studies, preeclamptic women had significantly higher blood lead levels as well as urinary lead extraction [14-16]. In this regard, a meta-analysis from 2018 concluded that the likelihood of a woman becoming preeclamptic increased by about $1.6 \%$ with every $1 \mu \mathrm{g} / \mathrm{dL}$ rise in their blood lead concentration [39]. Frequent monitoring of blood lead levels in pregnant women is unrealistic for our setting, but further research is needed to have a better understanding of the sources of pollution in Freetown. Beyond that, significantly more preeclamptic and eclamptic women drew their drinking water from a well or borehole, 
Table 3 Binary logistic regression model of predictors for PrE/E in PCMH

\begin{tabular}{|c|c|c|c|c|c|c|}
\hline Characteristics & Case n (\%) & Control n (\%) & COR $(95 \% \mathrm{Cl})$ & $p$-value & AOR $(95 \% \mathrm{Cl})$ & $p$-value \\
\hline \multicolumn{7}{|l|}{ Maternal assets } \\
\hline$\leq 50,000 \mathrm{SLL}$ & $82(40.0)$ & $87(19.7)$ & $2.72(1.89-3.92)$ & $<0.001$ & $2.56(1.63-4.02)$ & $<$ \\
\hline$>50,000 \mathrm{SLL}$ & $123(60.0)$ & $355(80.3)$ & 1.0 & & 1.0 & 0.001 \\
\hline \multicolumn{7}{|c|}{ Family predisposition for preeclampsia and eclampsia } \\
\hline Yes & $23(16.7)$ & $14(3.6)$ & $5.33(2.66-10.69)$ & $<0.001$ & $2.72(1.46-5.07)$ & 0.002 \\
\hline No & $115(83.3)$ & $373(96.4)$ & 1.0 & & 1.0 & \\
\hline \multicolumn{7}{|c|}{ Preexisting hypertension } \\
\hline Yes & $15(7.2)$ & $9(2.0)$ & $3.88(1.67-9.01)$ & 0.001 & $3.64(1.32-10.06)$ & 0.013 \\
\hline No & $193(92.8)$ & $449(98.0)$ & 1.0 & & 1.0 & \\
\hline \multicolumn{7}{|l|}{ UTI } \\
\hline Yes & 72 (33.6) & $73(16.0)$ & $2.66(1.82-3.88)$ & $<0.001$ & $2.02(1.28-3.19)$ & 0.002 \\
\hline No & $142(66.4)$ & $383(84.0)$ & 1.0 & & 1.0 & \\
\hline \multicolumn{7}{|c|}{ Prolonged diarrhoea } \\
\hline Yes & $72(33.6)$ & $55(12.0)$ & $3.71(2.49-5.53)$ & $<0.001$ & $2.81(1.63-4.86)$ & $<0.001$ \\
\hline No & $142(66.4)$ & $402(88.0)$ & 1.0 & & 1.0 & \\
\hline \multicolumn{7}{|l|}{ Malaria } \\
\hline Yes & $153(71.5)$ & $279(61.1)$ & $1.60(1.13-2.27)$ & 0.008 & $1.43(0.95-2.15)$ & 0.090 \\
\hline No & $61(28.5)$ & $178(38.9)$ & 1.0 & & 1.0 & \\
\hline \multicolumn{7}{|l|}{ MUAC } \\
\hline$>32 \mathrm{~cm}$ & $50(23.9)$ & $48(10.5)$ & $2.67(1.73-4.14)$ & $<0.001$ & $3.09(1.83-5.22)$ & $<0.001$ \\
\hline$\leq 32 \mathrm{~cm}$ & $159(76.1)$ & $408(89.5)$ & 1.0 & & 1.0 & \\
\hline \multicolumn{7}{|c|}{ Living close to a waste deposit } \\
\hline Yes & $59(29.4)$ & $70(16.3)$ & $2.14(1.44-3.18)$ & $<0.001$ & $1.94(1.15-3.25)$ & 0.013 \\
\hline No & $142(70.6)$ & $360(83.7)$ & 1.0 & & 1.0 & \\
\hline \multicolumn{7}{|c|}{ Well or borehole water as main source of drinking water } \\
\hline Yes & $61(28.9)$ & $80(17.6)$ & $1.90(1.30-2.79)$ & 0.001 & $2.05(1.31-3.23)$ & 0.002 \\
\hline No & $150(71.1)$ & $374(82.4)$ & 1.0 & & 1.0 & \\
\hline \multicolumn{7}{|c|}{ Average sleep duration } \\
\hline Short $<6 \mathrm{~h}$ & $139(70.2)$ & $247(54.9)$ & $1.94(1,36-2.77)$ & $<0.001$ & $1.48(0.96-2.29)$ & 0.078 \\
\hline Long $\geq 6 \mathrm{~h}$ & $59(29.8)$ & $203(45.1)$ & 1.0 & & 1.0 & \\
\hline \multicolumn{7}{|l|}{ Fruit intake } \\
\hline Inadequate & 75 (35.9) & $66(14.5)$ & $3.30(2.25-4.85)$ & $<0.001$ & $2.58(1.64-4.06)$ & $<0.001$ \\
\hline Adequate & $134(64.1)$ & 389 (85.5) & 1.0 & & 1.0 & \\
\hline
\end{tabular}

Binary Logistic Regression Model was calculated including variables matching criteria of a $p$-value $\leq 0.001$ and crude odds ratios $>1.5$. We additionally included the risk factor Malaria during pregnancy because of its previously described influence of Plasmodium falciparum infected placenta on PrE/E. Multiple Imputation was conducted with the Mersenne Twister as random number generator program and for generator initialization we chose the fixed value of $2,000,000$. We imputed 25 variables with 10 datasets. Statistical significance was set at $p$-value $<0.05$, crude and adjusted odds ratios and $95 \%$ confidence interval were calculated. The Hosmer Lemeshow goodness of fit test for logistic regression was applied and showed overall a good fit with a $p$-value $>0.05$

which doubled their risk for PrE/E. In 2019, WHO and United Nations Children's Fund published a report stating that in Sierra Leone only 10\% of the population had access to 'safely managed' drinking water services, defined as located inside the yard, available for at least $12 \mathrm{~h}$ per day and in line with standards regarding fecal and chemical contamination [34]. Nutritional factors also played an important role in our study, as an inadequate intake of fruits raised the risk for PrE/E almost threefold. In accordance, several studies from Ethiopia described a regular intake of fruits and vegetables to be protective against preeclampsia [22-25]. Interestingly, in contrast to those findings, the consumption of vegetables less than one time per week proved to be protective for $\mathrm{PrE} / \mathrm{E}$ in our analysis. This may again hint to environmental pollution exposure, following findings by Kim et al. (2016), who found vegetables to be one of the most lead loaded aliments affecting maternal health in the Republic of Korea [40]. Almost all of 
Table 4 Birth outcomes in PCMH

\begin{tabular}{|c|c|c|c|c|c|}
\hline Characteristics & Total n (\%) & Case Births n (\%) & Control Births n (\%) & COR $(95 \% \mathrm{Cl})$ & $p$-value \\
\hline Total & 713 & 229 & 484 & & \\
\hline Gestational age at delivery ${ }^{a}$ & 599 & 153 & 446 & & \\
\hline Median & $38(37-40)$ & $37(36-39)$ & $38(37-40)$ & & \\
\hline Means & $37.91( \pm 2.78)$ & $36.90( \pm 3.36)$ & $38.26( \pm 2.46)$ & & $<0.001$ \\
\hline Birth outcome & (653) & (169) & $(484)$ & & \\
\hline Live birth & $582(89.1)$ & $145(85.8)$ & $437(90.3)$ & 1.0 & \\
\hline Fresh stillbirth & $42(6.4)$ & $10(5.9)$ & $32(6.6)$ & $0.94(0.45-1.96)$ & 0.873 \\
\hline Macerated stillbirth & $29(4.4)$ & $14(8.3)$ & $15(3.1)$ & $2.81(1.33-5.97)$ & 0.005 \\
\hline Delivery mode & (653) & (169) & $(484)$ & & \\
\hline Vaginal spontaneous & $465(71.2)$ & $113(66.9)$ & $352(72.7)$ & 1.0 & \\
\hline Vaginal operative & $39(6.0)$ & $4(2.4)$ & $35(7.2)$ & $0.36(0.12-1.02)$ & 0.046 \\
\hline Caesarean section & $149(22.8)$ & $52(30.8)$ & $97(20.0)$ & $1.67(1.12-2.49)$ & 0.011 \\
\hline Preterm birth ${ }^{b}$ & (598) & (153) & $(445)$ & & \\
\hline Yes & $130(21.7)$ & $67(43.8)$ & $63(14.3)$ & $4.72(3.12-7.16)$ & $<0.001$ \\
\hline No & $468(78.3)$ & $86(56.2)$ & $382(85.7)$ & 1.0 & \\
\hline Birth weight ${ }^{c}$ & (543) & (132) & (411) & & \\
\hline$<2500 \mathrm{~g}$ & 75 (13.8) & $40(30.3)$ & $35(8.5)$ & $4.67(2.81-7.76)$ & $<0.001$ \\
\hline$\geq 2500 \mathrm{~g}$ & $468(86.2)$ & $92(69.7)$ & $376(91.5)$ & 1.0 & \\
\hline
\end{tabular}

Univariate analysis was conducted with Pearson's Chi-square test for categorical variables. Metric variables were compared using Independent T-Test and Mann-

Whitney-U-Test. Statistical significance was set at $p$-value $<0.05$, crude and adjusted odds ratios and $95 \%$ confidence interval were calculated

${ }^{a}$ gestational age at delivery; caesarean section included

${ }^{b}$ fetus born prior to the 37 th postmenstrual week; caesarean section included

${ }^{c}$ multifetal gestation excluded; caesarean section included

the aforementioned risk factors could be controlled through regular and frequent ANC visits with adequate screening and counseling. As a matter of fact, the National Protocols and Guidelines for Emergency Obstetric and Newborn Care recommend measuring blood pressure or performing urinalysis as well as counseling on nutrition, diet, and exercise at every single ANC visit [41]. However, in our sample, the average number of ANC visits until birth was far below the eight visits recommended by the National Protocols and Guidelines. One reason could be a general mistrust among pregnant women in health care facilities in Sierra Leone. This is mirrored in a recent qualitative study by Theuring et al. (2018) in the same hospital setting, which described some reluctance among pregnant women towards facility-based delivery care, suggesting that $\mathrm{PCMH}$ might need to improve communication skills of staff to build a trustful environment for their clients [42]. Sripad et al. (2019) supports this finding in their study from Nigeria, stating that respectful maternity care affects the willingness to seek care in ANC clients [43]. Above that, ANC services should be revised to offer more effective management of $\mathrm{PrE} / \mathrm{E}$ with extended risk factor assessment and management as well as education on early signs and symptoms of $\mathrm{PrE} / \mathrm{E}$ and if necessary, more frequent screenings for the condition. Regarding risk factor assessment and management, the skills of ANC staff might need to be widened towards a comprehensive strategy, including not only systematic screening of physical risk factors, but also intense counseling. This should comprise behavioral risk factors, like hygiene-related behavior to avoid infectious diseases presenting in UTIs or prolonged diarrhoea, and appropriate nutrition and diet counseling, including education on drinking water management. In this context, it would be worthwhile to focus on practical information empowering particularly those women lacking financial resources, for example by advising them how to cultivate fruits at home to improve their diet or teaching them how to obtain safe drinking water by boiling it and storing it correctly. The great poverty of the women in our setting must be apprehended as an underlying factor not only for low access to nutritious food and safe water supply but also for most other risk factors, including housing close to sources of pollution. In the case group, $40 \%$ of women had less than 50,000 SLL per month available to spend. In Nigeria, Sripad et al. found that the financial situation of women imposed a barrier of care-seeking in the early stages of PrE/E [43]. Health policymakers in Sierra Leone should, therefore, take into serious consideration how women with an identified high risk for a condition like PrE/E could benefit from reduced costs on healthy food, drinking water supply, and transportation to access healthcare services 
regularly, to enable ANC services to properly screen and manage clients at risk for PrE/E.

\section{Limitations}

The facility-based study design might entail a selection bias in the recruitment of the control group, because women who sought skilled delivery services or received inpatient treatment in the referral institution $\mathrm{PCMH}$ are likely to have experienced a more complicated pregnancy, e.g. with higher rates of multifetal gestations or with more severe complications during the current or previous pregnancy. Other limitations were low educational levels of participants, reduced diagnostic capacities due to the resource-limited setting, and lacking patient documentation. As a result, we had to face missing data as well as underreporting of e.g. reasons for previous pregnancy complications resulting in abortions or stillbirth as well as provided history of preexisting conditions.

\section{Conclusions}

$\mathrm{PrE} / \mathrm{E}$ is one of the leading causes of maternal mortality in Sierra Leone. Our findings suggest that systematic assessment of identified $\mathrm{PrE} / \mathrm{E}$ risk factors, including a family history of $\mathrm{PrE} / \mathrm{E}$, preexisting hypertension, or obesity by measuring the MUAC, should be performed early on in ANC, followed by continued close monitoring of potential behavioral risk factors throughout pregnancy to improve patient management. Counseling on nutrition, dietary aspects and exercise should be comprehensively addressed and be extended with practical information meaningful for the poorest parts of the population, such as on self-cultivation of fruits and achieving improved water safety. Regarding the high prevalence of UTIs and prolonged diarrhoea, improved hygiene-related behavior should also be strongly emphasized. Additionally, our study implies that further research on sources of environmental pollution in Freetown, especially regarding heavy metals like lead strain, is urgently needed.

\section{Abbreviations}

PrE/E: Preeclampsia and Eclampsia; PCMH: Princess Christian Maternity Hospital; UTIs: urinary tract infections; WHO: World Health Organization; DRC: Democratic Republic of Congo; MUAC: mid-upper arm circumflex; COR, AOR: crude and adjusted odds ratios; ANC: antenatal care; SLL: Sierra Leone Leones

\section{Supplementary Information}

The online version contains supplementary material available at https://doi. org/10.1186/s12884-021-03874-7.

Additional file 1. CASE GROUP Study Questionnaire. PDF (Adobe Acrobat) of the questionnaire used for data collection of cases.

Additional file 2. CONTROL GROUP Study Questionnaire. PDF (Adobe Acrobat) of the questionnaire used for data collection of controls.

\section{Acknowledgments}

We thank all study nurses, Annie Paris, Hawa Tina Bangura, Jeneba Conteh, Seibatu Bonnie and Mabinty, for their participation in data collection as well as all study participants who provided information. We thank CUAMM personnel, midwives and archive personnel from PCMH. We also thank Mr. Lewally, the partnership coordinator, and Alexander Hayes for proofreading. The study design and preliminary results were presented in a poster presentation: 'Assessing risk factors for preeclampsia and eclampsia in a main referral maternity hospital in Freetown, Sierra Leone', in the 11th European Congress on Tropical Medicine and International Health, Liverpool, UK, September $16-20,2019$

\section{Authors' contributions}

NS: data collection, data analysis, manuscript preparation, and revision; JS: study design, study preparation, data collection, manuscript revision; MK: study design, study preparation, manuscript revision; ST: concept development, study design, study preparation, overall supervision, manuscript preparation, and revision. All authors have read and approved the final manuscript.

\section{Funding}

This study was conducted with the support of the ESTHER-clinic partnership program of the German Agency for International Cooperation and the Federal Ministry for Economic Cooperation and Development, who did not influence the design of the study, on the collection, analysis, and interpretation of data, and on writing the manuscript. Open Access funding enabled and organized by Projekt DEAL.

\section{Availability of data and materials}

The data will not be publicly shared to protect the participants' anonymity. The anonymized data used for analysis can be made available upon request to the corresponding author at the Institute of Tropical Medicine and International Health, Charité-Universitätsmedizin Berlin, Germany.

\section{Declarations}

Ethics approval and consent to participate

Ethical clearance was provided from the Office of the Sierra Leone Ethics and Scientific Review Committee and the Ethical Committee of CharitéUniversitätsmedizin, Berlin, Germany (EA2/219/18). Participation in the study was completely voluntary. All the study participants were informed about the purpose of the study and written consent was obtained from each study participant. We obtained ethical approval to include pregnant minors from 15 years of age onwards if they gave written consent. Regarding minors below 15 years of age, we additionally obtained the consent of their present legal guardian.

\section{Consent for publication}

Not applicable.

\section{Competing interests}

The authors declare that they have no competing interests.

\section{Author details}

${ }^{1}$ Institute of Tropical Medicine and International Health,

Charité-Universitätsmedizin Berlin, corporate member of Freie Universität Berlin, Humboldt-Universität zu Berlin, and Berlin Institute of Health, Augustenburger Platz 1, 13353 Berlin, Germany. ${ }^{2}$ National School of Midwifery, Freetown, Sierra Leone. ${ }^{3}$ Princess Christian Maternity Hospital, Freetown, Sierra Leone.

Received: 25 August 2020 Accepted: 13 May 2021

Published online: 02 June 2021

\section{References}

1. Tranquilli AL, Dekker G, Magee L, Roberts J, Sibai BM, Steyn W, et al. The classification, diagnosis and management of the hypertensive disorders of pregnancy: a revised statement from the ISSHP. Pregnancy Hypertens. 2014; 4(2):97-104. https://doi.org/10.1016/j.preghy.2014.02.001.

2. Poon LC, Shennan A, Hyett JA, Kapur A, Hadar E, Divakar $H$, et al. The International Federation of Gynecology and Obstetrics (FIGO) initiative on 
pre-eclampsia: a pragmatic guide for first-trimester screening and prevention. Int J Gynaecol Obstet. 2019;145(Suppl 1):1-33. https://doi.org/1 $0.1002 /$ ijgo.12802.

3. Khan KS, Wojdyla D, Say L, Gulmezoglu AM, Van Look PF. WHO analysis of causes of maternal death: a systematic review. Lancet (London, England). 2006:367(9516):1066-74.

4. Abalos E, Cuesta C, Grosso AL, Chou D, Say L. Global and regional estimates of preeclampsia and eclampsia: a systematic review. Eur J Obstet Gynecol Reprod Biol. 2013;170(1):1-7. https://doi.org/10.1016/j.ejogrb.2013.05.005.

5. Duckitt K, Harrington D. Risk factors for pre-eclampsia at antenatal booking: systematic review of controlled studies. BMJ (Clinical research ed). 2005; 330(7491):565.

6. Cassell KA, O'Connell CM, Baskett TF. The origins and outcomes of triplet and quadruplet pregnancies in Nova Scotia: 1980 to 2001. Am J Perinatol. 2004;21(8):439-45. https://doi.org/10.1055/s-2004-835960.

7. Bartsch E, Medcalf KE, Park AL, Ray JG. Clinical risk factors for pre-eclampsia determined in early pregnancy: systematic review and meta-analysis of large cohort studies. BMJ (Clinical research ed). 2016;353:i1753.

8. Nilsson E, Salonen Ros H, Cnattingius S, Lichtenstein P. The importance of genetic and environmental effects for pre-eclampsia and gestational hypertension: a family study. BJOG. 2004;111(3):200-6. https://doi.org/1 0.1111/j.1471-0528.2004.00042x.X.

9. Bilano VL, Ota E, Ganchimeg T, Mori R, Souza JP. Risk factors of preeclampsia/eclampsia and its adverse outcomes in low- and middle-income countries: a WHO secondary analysis. PLoS One. 2014;9(3):e91198. https:// doi.org/10.1371/journal.pone.0091198.

10. Conde-Agudelo A, Villar J, Lindheimer M. Maternal infection and risk of preeclampsia: systematic review and metaanalysis. Am J Obstet Gynecol. 2008;198(1):7-22. https://doi.org/10.1016/j.ajog.2007.07.040.

11. Kiondo P, Wamuyu-Maina G, Bimenya GS, Tumwesigye NM, Wandabwa J, Okong P. Risk factors for pre-eclampsia in Mulago Hospital, Kampala Uganda. TM IH. 2012;17(4):480-7. https://doi.org/10.1111/j.1365-3156.2 011.02926.x

12. Ganchimeg T, Ota E, Morisaki N, Laopaiboon M, Lumbiganon P, Zhang J, et al. Pregnancy and childbirth outcomes among adolescent mothers: a World Health Organization multicountry study. BJOG. 2014;121(Suppl 1):408. https://doi.org/10.1111/1471-0528.12630.

13. Guerrier G, Oluyide B, Keramarou M, Grais RF. Factors associated with severe preeclampsia and eclampsia in Jahun, Nigeria. Int J Women's Health. 2013; 5(1):509-13.

14. Elongi Moyene JP, Scheers H, Tandu-Umba B, Haufroid V, Buassa-Bu-Tsumbu $B$, Verdonck F, et al. Preeclampsia and toxic metals: a case-control study in Kinshasa, DR Congo. Environ/ Health. 2016;15(1):48. https://doi.org/10.1186/ s12940-016-0132-1.

15. Musa Obadia P, Kayembe-Kitenge T, Haufroid V, Banza Lubaba Nkulu C, Nemery B. Preeclampsia and blood lead (and other metals) in Lubumbashi, DR Congo. Environ Res. 2018;167:468-71. https://doi.org/10.1016/j.envres.2 018.07.032.

16. Ikechukwu IC, Ojareva OI, Ibhagbemien AJ, Okhoaretor OF, Oluwatomi OB, Akhalufo OS, et al. Blood lead, calcium, and phosphorus in women with preeclampsia in Edo state, Nigeria. Arch Environ Occup Health. 2012;67(3): 163-9. https://doi.org/10.1080/19338244.2011.619212.

17. Anorlu RI, Iwuala NC, Odum CU. Risk factors for pre-eclampsia in Lagos, Nigeria. Aust N Z J Obstet Gynaecol. 2005;45(4):278-82. https://doi.org/1 0.1111/j.1479-828X.2005.00411.X.

18. Owusu JT, Anderson FJ, Coleman J, Oppong S, Seffah JD, Aikins A, et al. Association of maternal sleep practices with pre-eclampsia, low birth weight, and stillbirth among Ghanaian women. Int J Gynaecol Obstet. 2013; 121(3):261-5. https://doi.org/10.1016/j.jijo.2013.01.013.

19. Adam I, Elhassan EM, Mohmmed AA, Salih MM, Elbashir MI. Malaria and preeclampsia in an area with unstable malaria transmission in Central Sudan. Malar J. 2011;10(1):258. https://doi.org/10.1186/1475-2875-10-258.

20. Ndao CT, Dumont A, Fievet N, Doucoure S, Gaye A, Lehesran JY. Placental malarial infection as a risk factor for hypertensive disorders during pregnancy in Africa: a case-control study in an urban area of Senegal, West Africa. Am J Epidemiol. 2009;170(7):847-53. https://doi.org/10.1093/aje/kwp207.

21. Sartelet H, Rogier C, Milko-Sartelet I, Angel G, Michel G. Malaria associated pre-eclampsia in Senegal. Lancet (London, England). 1996;347(9008):1121.

22. Grum T, Hintsa S, Hagos G. Dietary factors associated with preeclampsia or eclampsia among women in delivery care services in Addis Ababa, Ethiopia: a case control study. BMC Res Notes. 2018;11(1):683. https://doi.org/10.1186/ s13104-018-3793-8.

23. Endeshaw M, Abebe F, Bedimo M, Asart A. Diet and pre-eclampsia: a prospective multicentre case-control study in Ethiopia. Midwifery. 2015; 31(6):617-24. https://doi.org/10.1016/j.midw.2015.03.003.

24. Endeshaw M, Abebe F, Worku S, Menber L, Assress M, Assefa M. Obesity in young age is a risk factor for preeclampsia: a facility based case-control study, Northwest Ethiopia. BMC Pregnancy Childbirth. 2016;16(1):237. https://doi.org/10.1186/s12884-016-1029-2.

25. Mekie M, Mekonnen W, Assegid M. Cohabitation duration, obstetric, behavioral and nutritional factors predict preeclampsia among nulliparous women in West Amhara zones of Ethiopia: age matched case control study. PLoS One. 2020;15(1):e0228127. https://doi.org/10.13 71/journal.pone.0228127.

26. Mahomed K, Williams MA, Woelk GB, Jenkins-Woelk L, Mudzamiri S, Longstaff $L$, et al. Risk factors for pre-eclampsia among Zimbabwean women: maternal arm circumference and other anthropometric measures of obesity. Paediatr Perinat Epidemiol. 1998;12(3):253-62. https://doi.org/10.1 046/j.1365-3016.1998.00116.x.

27. Statistics Sierra Leone. Population size and distribution. In: Sierra Leone 2015 Population and Housing Census National Analytical Report. Statistics Sierra Leone. 2017. [https://www.statistics.sl/images/StatisticsSL/Documents/Census/2 015/2015_census_national_analytical_report.pdf. Accessed 20 June 2020.]

28. World Health Organization: Annex 17. Trends in estimates of maternal mortality ratio (MMR, maternal deaths per 100000 live births), by country and territory, 2000-2017. In: Trends in maternal mortality 2000 to 2017: estimates by WHO, UNICEF, UNFPA, World Bank Group and the United Nations Population Division. 1 edn. Edited by World Health Organization. Geneva: World Health Organization; 2019: p.103.

29. Government of Sierra Leone Ministry of Health \& Sanitation. Causes of Maternal Deaths. In: Maternal Death Surveillance \& Response, Annual Report 2016. 2017 [https://www.afro.who.int/sites/default/files/2017-06/mdsrreport. pdf. Accessed 20 June 2020.]

30. Vousden N, Lawley E, Seed PT, Gidiri MF, Goudar S, Sandall J, et al. Incidence of eclampsia and related complications across 10 low- and middle-resource geographical regions: secondary analysis of a cluster randomised controlled trial. PLoS Med. 2019;16(3):e1002775. https://doi. org/10.1371/journal.pmed.1002775.

31. Princess Christian Maternity Hospital: Annual report of 2018. In. Freetown; 2019.

32. Government of Sierra Leone Ministry of Health and Sanitation. National Protocol for Management of Hypertensive Disorders of Pregnancy and Eclampsia. In: National Protocols and Guidelines for Emergency Obstetric and Newborn Care. Freetown: Government of Sierra Leone Ministry of Health and Sanitation; 2018. p. 22-3.

33. Okereke CE, Anyaehie UB, Dim CC, lyare EE, Nwagha UI. Evaluation of some anthropometric indices for the diagnosis of obesity in pregnancy in Nigeria: a cross-sectional study. Afr Health Sci. 2013;13(4):1034-40. https://doi.org/1 0.4314 /ahs.v13i4.25.

34. United Nations Children's Fund (UNICEF) and World Health Organization. Reducing inequalities in safely managed services. In: United Nations Children's Fund (UNICEF) and World Health Organization, editor. Progress on household drinking water, sanitation and hygiene 2000-2017 Special focus on inequalities. 1st ed. New York: United Nations Children's Fund (UNICEF) and World Health Organization; 2019. p. 48.

35. Bandoli G, Lindsay S, Johnson DL, Kao K, Luo Y, Chambers CD. Change in paternity and select perinatal outcomes: causal or confounded? J Obstet Gynaecol. 2012;32(7):657-62. https://doi.org/10.3109/01443615.2012.698669.

36. Sibai B, Dekker G, Kupferminc M. Pre-eclampsia. Lancet (London, England). 2005:365(9461):785-99.

37. von Dadelszen $P$, Magee LA. Could an infectious trigger explain the differential maternal response to the shared placental pathology of preeclampsia and normotensive intrauterine growth restriction? Acta Obstet Gynecol Scand. 2002:81(7):642-8. https://doi.org/10.1080/j.1600-0412.2002.810710.x.

38. Kaduma J, Seni J, Chuma C, Kirita R, Mujuni F, Mushi MF, et al. Urinary tract infections and preeclampsia among pregnant women attending two hospitals in Mwanza City, Tanzania: a 1:2 matched case-control study. Biomed Res Int. 2019;2019:3937812.

39. Poropat AE, Laidlaw MAS, Lanphear B, Ball A, Mielke HW. Blood lead and preeclampsia: a meta-analysis and review of implications. Environ Res. 2018; 160:12-9. https://doi.org/10.1016/j.envres.2017.09.014. 
40. Kim JH, Lee SJ, Kim SY, Choi G, Lee JJ, Kim HJ, et al. Association of food consumption during pregnancy with mercury and lead levels in cord blood. Sci Total Environ. 2016;563-564:118-24. https://doi.org/10.1016/j.scitotenv.2 016.04.082.

41. Government of Sierra Leone Ministry of Health and Sanitation. National Guideline for Antenatal Care. In: National Protocols and Guidelines for Emergency Obstetric and Newborn Care. Freetown: Government of Sierra Leone Ministry of Health and Sanitation; 2018. p. 2-3.

42. Theuring S, Koroma AP. Harms G: "in the hospital, there will be nobody to pamper me": a qualitative assessment on barriers to facility-based delivery in post-Ebola Sierra Leone. Reprod Health. 2018;15(1):155. https://doi.org/1 0.1186/s12978-018-0601-9.

43. Sripad P, Kirk K, Adoyi G, Dempsey A, Ishaku S, Warren CE. Exploring survivor perceptions of pre-eclampsia and eclampsia in Nigeria through the health belief model. BMC Pregnancy Childbirth. 2019;19(1):431. https://doi.org/1 0.1186/s12884-019-2582-2.

\section{Publisher's Note}

Springer Nature remains neutral with regard to jurisdictional claims in published maps and institutional affiliations.

Ready to submit your research? Choose BMC and benefit from:

- fast, convenient online submission

- thorough peer review by experienced researchers in your field

- rapid publication on acceptance

- support for research data, including large and complex data types

- gold Open Access which fosters wider collaboration and increased citations

- maximum visibility for your research: over $100 \mathrm{M}$ website views per year

At BMC, research is always in progress.

Learn more biomedcentral.com/submissions 